\title{
Correction to: Responses of Lotus corniculatus to environmental change. 4: Root carbohydrate levels at defoliation and regrowth climatic conditions are major drivers of phenolic content and forage quality
}

\author{
Phillip Morris $^{1}$ - Eunice B. Carter ${ }^{1,2} \cdot$ Barbara Hauck $^{1,2}$. John-Wayne Hughes ${ }^{1} \cdot$ Gordon Allison $^{1,2}$. \\ Michael K. Theodorou ${ }^{1,3}$
}

Published online: 15 April 2021

(c) Springer-Verlag GmbH Germany, part of Springer Nature 2021

\section{Correction to: Planta (2021) 253:38}

https://doi.org/10.1007/s00425-020-03523-x

The original version of this article unfortunately contained a mistake. The co-author Michael K. Theodorou was not listed among the authors or in the author contribution statement and an additional person was missed from the acknowledgments section. The original article has been corrected.

Author contribution statement PM and MKT devised the project and acquired the GERP grant. MKT and EBC devised the digestibility protocol. EBC produced experimental material and carried out sugar, tannin and digestibility analysis. JWH. GA and BH carried out the flavonoid and lignin analysis. PM wrote and GA edited the manuscript.

Acknowledgements We would like to thank Julie Downsborough, Delma Jones and Alison Brooks for technical and analytical help. This research was supported by the BBSRC under the Global Environment Response Programme, (GERP Grant number PG230/526), and BBSRC strategic grants to IGER (BBS/E/G/000033073120, 3390 and PU15), and the authors have no conflict of interest to declare.

The original article can be found online at https://doi.org/10.1007/ s00425-020-03523-x.

Phillip Morris

morrisp14@hotmail.co.uk

1 Institute of Grassland and Environmental Research (IGER), Plas Gogerddan, Aberystwyth, Ceredigion SY23 3EB, UK

2 Institute of Biological, Environmental and Rural Sciences, Aberystwyth University, Plas Gogerddan, Aberystwyth, Ceredigion SY23 3EB, UK

3 Department of Agriculture and Environment, Agriculture Centre for Sustainable Energy Systems, Harper Adams University, Newport, Shropshire TF10 8NB, UK
Publisher's Note Springer Nature remains neutral with regard to jurisdictional claims in published maps and institutional affiliations. 